\title{
DISFUNCIÓN ERÉCTIL DE ORIGEN NEUROLÓGICO
}

\author{
Antonio Sánchez Ramos, Juan Antonio Godino Durán' y Antonio Oliviero'.
}

Servicio de Rehabilitación y Sección de Neurología y Neurofisiología Clínica'. Hospital Nacional de Parapléjicos. SESCAM. Toledo. España.

\begin{abstract}
Resumen.- La disfunción eréctil (DE) de causa neurológica, se produce como consecuencia de las alteraciones de las vías nerviosas tanto autonómicas como somáticas o la combinación de ambas y de los componentes cerebrales que inducen la erección. Esta revisión intenta explicar los mecanismos fisiopatológicos de las más frecuentes alteraciones neurológicas que causan disfunción eréctil y trastornos sexuales.
\end{abstract}

Palabras clave: Lesión medular. Esclerosis múltiples. Neurofisiología. Potenciales evocados. Disfunción eréctil.

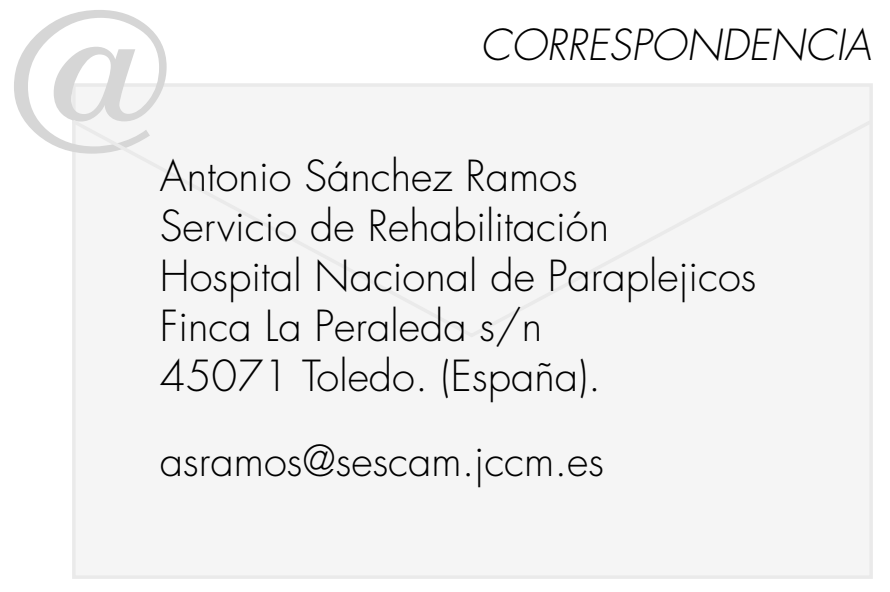

Summary.- Neurogenic erectile dysfunction is a consequence of alterations in neural pathways, autonomic, somatic, the combination of both or brain components that induce erection. This review aims to explain the physiopathological mechanisms of the most frequent neurological alterations causing erectile dysfunction and sexual disorders.

Keywords: Erectile dysfunction. Spinal cord. Multiple sclerosis. Neurophysiology. Evoked potentials.

\section{INTRODUCCIÓN}

La erección del pene es una respuesta fisiológica en la que participan estructuras neurológicas, vasculares, endocrinas e interacciones psicológicas. Los nervios del pene y las eferencias y aferencias del sistema nervioso central y periférico garantizan el correcto funcionamiento y la sincronización necesaria para una erección fisiológica eficaz. La estimulación sensitiva y local de los genitales provoca la erección refleja mediadas por el centro parasimpático sacro (S2-S3) de la médula espinal y los estímulos psicógenos percibidos, provocan la erección que podemos llamar cerebral o psíquica en las que están implicados el rinencéfalo, los núcleos talámicos y estructuras límbicas, siendo estos mensajes integrados en la región preóptica medial del hipotálamo (1).

Funcionalmente el nervio pélvico es el fundamental para provocar una erección y del efecto contrario (la detumescencia) es responsable el simpático paravertebral.

La Disfunción Eréctil (DE) de causa neurológica, se produce como consecuencia de las altera- 
ciones de las vías nerviosas tanto autonómicas como somáticas o la combinación de ambas y de los componentes cerebrales que inducen la erección $(2,3)$.

La inervación somática es fácilmente valorable por estudios neurofisiológicos, pero la inervación autonómica es mucho más compleja y no ha sido siempre bien interpretada.

Virtualmente lesiones en cualquier punto de las conexiones nerviosas entre el cerebro y el pene, pueden afectar a los mecanismos fisiológicos de esta función. Las causas de DE por afectación nerviosa son variadas, siendo las vías nerviosas centrales tanto ascendentes como descendentes y los nervios periféricos, los que pueden verse afectados por múltiples causas como son las traumáticas, inflamatorias, vasculares, degenerativas, metabólicas, tumorales, compresivas o iatrogénicas, entre otras (3).

Es indudable el reconocido papel de órgano centinela como predictor de muchas patologías neurológicas que permitirían un diagnóstico precoz.

El objetivo de este trabajo es el de hacer un acercamiento fisiopatológico de las diferentes estructuras neuroandrológicas que se ven afectadas cuando se produce un problema de DE de origen neurológico.

\section{FISIOPATOLOGÍA DE LA DISFUNCIÓN ÉRECTIL}

Cualquier alteración o enfermedad que afecte a las vías nerviosas tanto a nivel de los nervios periféricos, medular, de la cauda equina y cerebral pueden provocar disfunción eréctil.

Describiremos los principales mecanismos fisiopatológicos de la DE en este orden.

\section{Neuropatías}

La innervación de los órganos genitales es somática y autonómica. El control periférico de la erección se lleva acabo por fibras nerviosas somáticas (aferente sensitiva del nervio pudendo y eferente motora del mismo nervio), fibras nerviosas parasimpáticos (nervio pélvico) y fibras nerviosas simpáticas (nervio hipogástrico) que actúan de manera coordinada.

Respecto a la erección hay que considerar dos aspectos fundamentales que implican los nervios periféricos: las aferencias somáticas que trasladan la información táctil (estímulo sexual) y las eferencias parasimpáticas. Estas últimas producen el aumento de flujo hemático en las arteriolas peneanas y el rela- jamiento de la musculatura lisa. Al final de este proceso se produce la tumescencia y la rigidez del pene (erección) $(1,2)$.

La afectación del sistema nervioso periférico, que puede producir disfunción eréctil, puede deberse a enfermedades de tipo sistémico o a traumatismos (accidentales/ yatrogénicos en pelvis-región lumbosacra).

Las enfermedades sistémicas más frecuentes causantes de disfunción eréctil son la diabetes, alcoholismo, enfermedades carenciales (déficit vitamínico, tóxicos, etc.).

Parecería obvio pensar que en estas enfermedades las causas de la disfunción eréctil sea exclusivamente el malfuncionamiento de los nervios. En realidad las bases fisiopatológicas no son tan claras.

Por ejemplo, en los cuerpos cavernosos de los diabéticos con disfunción eréctil se observa una disminución del contenido de noradrenalina y una disminución de la síntesis de acetilcolina. Esta última con una buena correlación con la duración de la enfermedad. Estas alteraciones podrían estar relacionadas con una disfunción nerviosa. Independiente lo menos dependiente) de la función nerviosa periférica sería la degeneración nitrérgica observada en la diabetes. La inactivación excesiva y patológica del óxido nítrico podría ser un efecto directo de la hiperglicemia. Además se observa una destrucción del tejido de los cuerpos cavernosos por mecanismo de apoptosis, cuyo mecanismo no es del todo claro (4).

Estos múltiples mecanismos que pueden intervenir en la $D E$ en la diabetes pueden explicar la falta de correlación entre los estudios neurofisiológicos y la presencia y severidad de la DE. En cualquier caso merece la pena recordar que alteraciones funcionales en nervios periféricos evaluados con estudio del reflejo bulbocavernoso y uretro-anal y con el estudio de potenciales evocados del nervio pudendo son más frecuentes en diabéticos con $D E$ que en diabéticos sin DE. Esta consideración es importante puesto que no hay consenso sobre si el estudio neurofisiológico de miembros inferiores normalmente utilizado en el diagnóstico de neuropatía diabética es suficiente para la valoración de paciente diabético con $\mathrm{DE}$.

Uno de los mecanismos fisiopatológicos responsables de la DE en neuropatías es la desaferentación/deseferentación producida por el daño al nervio. Los estudios neurofisiológicos pueden documentar una disminución de la amplitud de los potenciales de nervio sensitivo (ej.: pudendo) y de los potenciales de acción motor compuesto (ej.: bulbocavernoso) (4). 
Los estudios con EMG podrán demostrar los signos de denervación aguda y potenciales de unidad motora de tipo reinervativo, confirmando la alteración del nervio y dando informaciones sobre la cronicidad del proceso patológico (4).

\section{Mielopatias y lesiones de la cauda equina}

Un $80 \%$ de varones con $L M$ consigue algún tipo de erección bien sean de características reflejas, voluntarias o mixtas, pero generalmente suelen ser insuficientes (a veces en rigidez y sobre todo en tiempo) para conseguir relaciones coitales satisfactorias (5).

No todas las lesiones medulares son iguales. Según la clasificación neurológica de la (ASIA) American Spinal Injury Asociation, las lesiones medulares pueden ser completas (grado A) y lesiones incompletas grados $B, C$ y $D$. Además estas lesiones pueden ser diferentes por el nivel metamérico de afectación (cervicales, dorsales, lumbares y sacras), así como lesiones transversas o longitudinales.

Virtualmente las mielopatias de cualquier causa pueden producir disfunción eréctil, utilizaremos el modelo de la lesión medular completas, que permite describir la lesiones a diferentes niveles de manera más comprensible.

La erección de tipo refleja se provoca por estímulos sensitivos en los genitales generando un arco reflejo que lleva el impulso nervioso desde ellos a la médula espinal, (al centro parasimpático sacro S2-S4) y vuelve hacia el pene, condicionando una erección refleja (6-8).

En las LM completas (Figura 1), desde el punto de vista neurofisiopatológico, cuando la lesión asienta por encima del segmento medular D10 sin estar destruidos los centros del control simpático y parasimpático y una vez superado el período de shock medular en la fase aguda de la lesión, el paciente consigue erecciones de características reflejas con estímulos manuales o roces en el pene (arco reflejo medular).

Si la lesión destruye los segmentos D1 1-L2, es donde se observa el mayor número de casos de disfunción eréctil grave, aunque también pueden observarse erecciones reflejas (si el centro parasimpático no está destruido) y/o psicógenas, en función del nivel de la lesión y la extensión de las metámeras medulares afectadas $(1,2)$.

Cuando la lesión se sitúa en los segmentos lumbosacros (L3-S5), el paciente puede conseguir erecciones al parecer provocadas por las fibras ge- neradoras de erección que el nervio hipogástrico, vía que se convierte en erectogénica cuando existe lesión del centro parasimpático sacro o de las vías que de éste parten hacia el plexo pélvico. Este es un mecanismo compensatorio como consecuencia de una reorganización anatómica de las vías erectogénicas cuya configuración anatómica está todavía por descifrar, pero que vemos con harta frecuencia en los pacientes lesionados con este nivel de lesión $(1,2)$. De todas formas estas erecciones serán de menor rigidez y en general más difíciles de mantener.

También podemos observar en estas lesiones que tienen destruido el centro parasimpático sacro, una disfunción completa con impotencias completas (que no responden al tratamiento con PGEl) y que pueden presentar apoptosis de células endoteliales por desaferentación, al igual que se han evidenciado en paciente con prostatectomía radical o en diabetes.

En LM incompleta la respuesta varía según cada paciente. En los que tienen preservados los centros de la erección y eyaculación, con frecuencia los factores psicológicos pueden influir de forma negativa en su capacidad de respuesta sexual.

Fisiopatología de la DE. Lesión medular

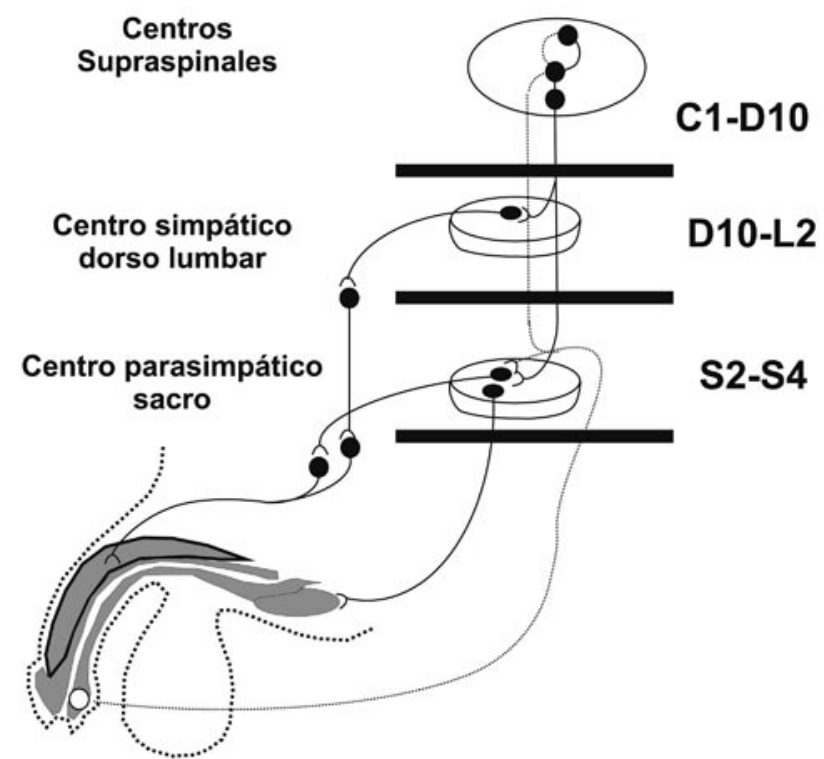

FIGURA 1. Fisiopatología de la disfunción eréctil en lesiones completas de la medula espinal: Esquema de los centros supraespinales y medulares implicados en el mecanismo de la erección. Diferentes niveles de lesión medular producen cuadros clínicos y neurofisiológicos característicos. 
Resumiendo, la fisiopatología de la DE en lesión medular dependerá del compromiso de las vías nerviosa centrales que conectan el cerebro con los centros medulares responsables de la erección y de la eyaculación. Estas alteraciones son clínicamente importante en pacientes con lesiones por encima del nivel medular D10 (si la médula conserva su indemnidad por debajo de la lesión) característicamente presentarán indemnidad del centro medular simpático y el centro sacro. Desde el punto de vista neurofisiológico esta integridad anatómica se reflejará en una normalidad del reflejo bulbocavernoso (Figura 2) y del estudio electromiográfico del músculo bulbocavernosos. Tal vez esta valoración neurofisiológica sea importante porque la lesión medular por encima del nivel D10 dificulta grandemente la valoración clínica de los niveles medulares más distales. La alteración de las vías ascendentes y descendentes medulares será fácilmente documentada clínicamente. Una alteración neurofisiológica de las vías ascendentes se reflejará en los potenciales evocados somatosensoriales obtenidos por estimulación de los nervios de miembros inferiores y por estimulación del nervio pudendo. Una alteración neurofisiológica de las vías descendentes (corticoespinal) se reflejará en los potenciales evocados motores obtenidos por estimulación magnética transcraneal registrando la respues-

\section{Estudio del reflejo bulbocavernoso.}

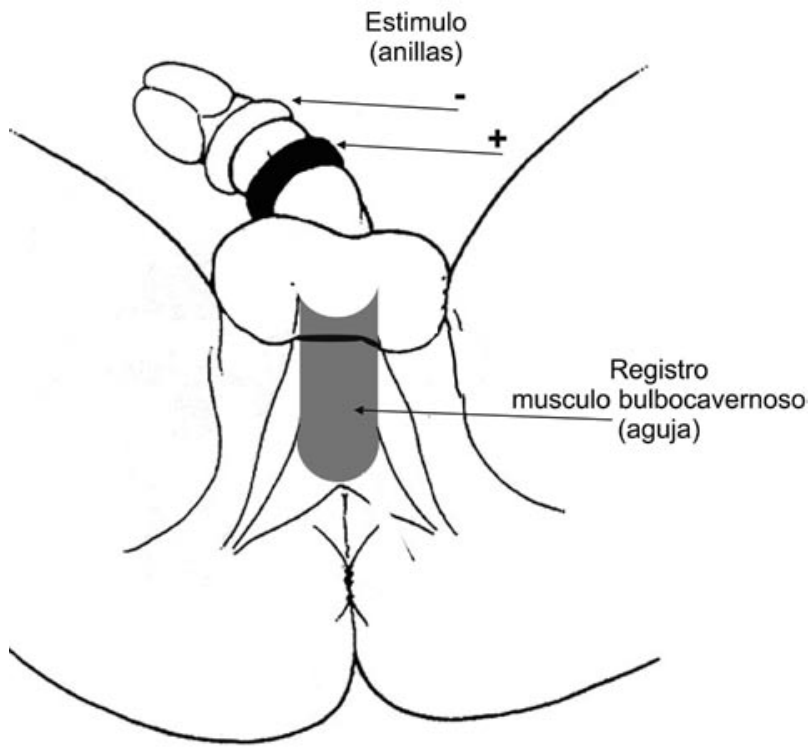

FIGURA 2. Estudio neurofisiológico del reflejo bulbocavernoso: Método de Tackmann lestimulación con electrodo de anilla en pene y registro con aguja concéntrica en músculo bulbocavernosol. ta evocada en los músculos de miembros inferiores y en músculo bulbocavernoso $(9,10)$. La respuesta cutánea simpática también confirmará la alteración de las vías descendentes puesto que será normal por encima de la lesión y alterada en su registro a nivel genital. Es importante recordar que estas alteraciones neurofisiológicas son independiente de la alteración del centro medular simpático (4).

Las lesiones medulares completas entre D10L2 (si hay indemnidad de las estructuras nerviosas por debajo de la lesión) tendrán una gran afectación del centro simpático medular mientras el centro sacro estará indemne. La indemnidad del centro sacro se podrá documentar clínica y neurofisiologicamente por estar preservado el reflejo bulbocavernoso y por no presentar alteraciones el estudio electromiográfico del músculo bulbocavernoso $(1,2,4)$. Las vías largas motoras (corticoespinales) y somatosensoriales (cordones posteriores) se verán claramente afectadas (9-11). Esta afectación será evidenciable clínica y neurofisiologicamente (potenciales evocados motores y sensoriales) de manera similar a lo reportado anteriormente por las lesiones por encima del nivel medular D10. A diferencia de las lesiones medulares con nivel por encima de D10 se pueden observar en la componente simpático cutánea que dependerá del número de metámeras afectadas a nivel D10-L2. La respuesta cutánea simpático refleja siempre es normal a nivel de la palma de la mano con este nivel de lesión. Al contrario, a nivel genital cuanto más próxima al nivel D10 tanto más probablemente esta valoración documentará alteraciones. Las lesiones medulares completas con afectación del centro sacro S2-S4 se presentan con unas características peculiares y son probablemente las lesiones que producen una disfunción eréctil más severa y de mayor complejidad en el tratamiento. Estas lesiones se caracterizan por la destrucción de las motoneuronas del músculo bulbocavernoso. Esto se refleja en la pérdida del reflejo bulbocavernoso y la denervación (evidente el la fase aguda de lesión) del músculo bulbocavernos (4).

También característica es la alteración de las vías largas. Los potenciales evocados motores obtenido en músculo bulbocavernoso y los potenciales evocados somatosensoriales por estimulación del nervio pudendo serán anormales mientras las mismas técnicas no serán siempre alteradas en miembros inferiores (la mayoría de los músculos y nervios de miembros inferiores que se valoran con estas técnicas están por encima del nivel S2) (10).

La fisiopatología de la disfunción eréctil producida por las lesiones de cola de caballo es parecida a las de las neuropatías (la alteración de las fibras 
que constituyen los nervios periféricos en el caso de la cola de caballo es muy proximal y frecuentemente muy heterogénea). La valoración neurofisiológica también es compleja y dependiente del tamaño de la lesión y de las raíces involucradas. El grado de afectación de estos pacientes es muy variable y va desde los grados mínimos de disfunción eréctil hasta los casos severos, éstos últimos se comportan de manera similar a los pacientes con afectación severa medular del centro sacro $(1,2)$.

El modelo de la lesión medular completa nos ha permitido describir los principales mecanismos que producen los diferentes cuadros de disfunción eréctil. Desde un punto de vista más propiamente clínico hay que considerar que en las lesiones medulares incompletas y en las mielopatías de origen no traumáticas (inflamatorias, degenerativas, vasculares, etc.) los cuadros clínicos y neurofisiológicos son mucho más variados $(3,5-8)$.

\section{Cerebropatías}

Muchas de la actividad refleja de la erección esta localizada en medula espinal. Sin embargo muchísimas regiones cerebrales están involucradas en aspectos muy importantes de la función sexual. Por ejemplo muchas neuronas hipotalámicas tienen conexiones con las regiones espinales responsables de la erección. Estas vías hipotálamo-espinales pasan en el funículo dorsolateral. También los núcleos paraventriculares tienen conexiones con los centros espinales. Además de estas vías, más o menos directas, hay que considerar que en el cerebro se localiza todo el control del eje hipotálamo-hipófisis-gonadal y que muchas áreas cerebrales son fundamentales en el correcto funcionamiento de funciones como el deseo sexual, la excitación y el mantenimiento de la excitación sexual y de la especificidad de la respuesta sexual. A nivel molecular hay que destacar el papel de los circuitos dopaminergicos y serotoninergico en relación al deseo sexual. Las patologías cerebrales que interfieran con cada una de estas áreas puede tener un efecto sobre la función eréctil y sobre el comportamiento sexual $(1,2)$.

Las alteraciones endocrinológicas producidas por disfunción del eje hipotálamo-hipófisis se presentan sobretodo con un disminución del deseo sexual y esto sería una causa directa de los efectos de la alteración de la regulación hormonal (ej.: aumento prolactina, disminución testosterona, etc.).

Entre las enfermedades que más frecuentemente afectan el cerebro hay que considerar los traumatismos craneoencefálicos y las vasculopatías cerebrales. En estas enfermedades es muy frecuente la alteración del comportamiento sexual y la disfunción eréctil es frecuentemente de origen psicógena.

La DE y los trastorno del comportamiento sexual son mucho más frecuentes en las lesiones frontales y temporales respecto a las lesiones parietales y occipitales. Las conexiones entre estructuras cerebrales anteriores y comportamiento sexual son numerosas y la sintomatología es muy variada dependiendo de la extensión, la localización y la bilateralidad de las lesiones (11). Los síntomas más frecuentes son disminución de la libido, DE y eyaculación retrasada. Menos frecuentes pero no menos importantes son los casos de aumento de la libido, la hipersexualidad y la pansexualidad producida por lesiones cerebrales. Por ejemplo las lesiones temporales anteriores bilaterales producen una hipersexualidad marcada (Sindrome de Kluver-Busy) (11).

En las afectaciones cerebrales merecen unas consideraciones, en especial los trastornos del movimiento. La enfermedad de Parkinson (EP), por ejemplo, presenta dos tipos de alteraciones interesantes desde un punto de vista fisiopatológico. Los efectos de la enfermedad produce una disfunción eréctil en hasta el $50 \%$ de los pacientes parkinsonianos y además las dificultades motoras y el temblor (que aumenta durante el coito), reducen la posibilidad de un sexualidad satisfactoria (12). La causa de la DE

Estudio del reflejo bulbocavernoso.

\section{Normal Patológico}

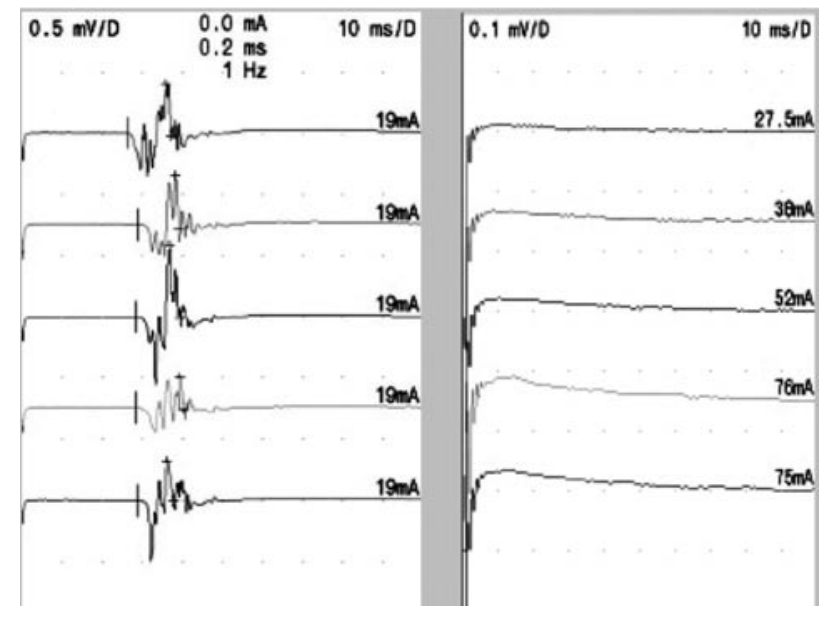

FIGURA 3. Estudio neurofisiológico del reflejo bulbocavernoso: Ejemplo de registro en condiciones normales y patológicos. 
TABLA I. ESTUDIOS NEUROFISIOLOGICOS DEL CENTRO MEDULAR SACRO Y DEL CENTRO SIMPATICO.

1. Estudio neurofisiológico del reflejo bulbocavernoso (RB).

2. Estudio electromiográfico del músculo bulbocavernoso.

3. Estudio de potenciales evocados somatosensoriales (PES) del nervio pudendo.

4. Estudio de neurografía sensitiva del nervio dorsal del pene.

5. Estudio de neurografía motora del nervio pudendo.

6. Estudio de estimulación magnética transcraneal con registro en músculo bulbocavernoso (MB).

7. Estudio cuantitativo de la sensibilidad (QST).

en EP no esta clara pero se considera una alteración autonómica asociada a la enfermedad. Los parkinsonianos no sufren sólo este problema directo de la enfermedad también el tratamiento dopaminérgico genera problemas del comportamiento sexual. Son frecuentes los casos de cambios de la sexualidad asociados al tratamiento dopaminérgico: hipersexualidad, pansexualidad, desinhibición sexual, etc. Si

TABLA II.

\begin{tabular}{|c|c|c|c|c|c|c|c|}
\hline & $\begin{array}{c}\text { Reflejo } \\
\text { Bulbocavernoso }\end{array}$ & $\begin{array}{c}\text { EMG } \\
\text { Bulbocavernoso }\end{array}$ & $\begin{array}{l}\text { PESS N } \\
\text { Pudendo }\end{array}$ & \begin{tabular}{|c|} 
TMS \\
Bulbocavernoso
\end{tabular} & \begin{tabular}{|} 
NSN \\
Dorsal pene
\end{tabular} & $\begin{array}{c}\text { NMN } \\
\text { Pudendo }\end{array}$ & $\begin{array}{c}\text { Respuesta } \\
\text { simpático refleja }\end{array}$ \\
\hline $\begin{array}{l}\text { LMC ENCl- } \\
\text { MA DIO }\end{array}$ & Presente & $\begin{array}{c}\text { Ausencia } \\
\text { denervación }\end{array}$ & Alterado & Alterado & Normal & Normal & $\begin{array}{l}\text { Presente palma } \\
\text { Ausente genital }\end{array}$ \\
\hline $\begin{array}{c}\text { LMC ENTRE } \\
\text { D10-L2 }\end{array}$ & Presente & $\begin{array}{c}\text { Ausencia } \\
\text { denervación }\end{array}$ & Alterado & Alterado & Normal & Normal & $\begin{array}{l}\text { Presente palma } \\
\text { Presente/Ausente } \\
\text { genital }\end{array}$ \\
\hline $\begin{array}{l}\text { LMC } \\
\text { S2-S4 }\end{array}$ & Ausente & Denervación * & Alterado & Alterado & Normal & $\begin{array}{l}\text { Ausente o } \\
\text { amplitud }\end{array}$ & $\begin{array}{l}\text { Presente palma } \\
\text { Presente genital }\end{array}$ \\
\hline LCC & $\begin{array}{l}\text { Presente- } \\
\text { Abolido- } \\
\text { Retrasado } \\
\text { ipsi/bil-* * }\end{array}$ & $\begin{array}{l}\text { Presencia- } \\
\text { ausencia de- } \\
\text { nervación ** }\end{array}$ & (Alterado) 1 & Alterado** & Normal & Alterada** & $\begin{array}{l}\text { Presente palma } \\
\text { Presente genital }\end{array}$ \\
\hline
\end{tabular}

EMG: electromiografía, PESS N: potenciales evocados somatosensoriales de Nervio, TMS: estimulación magnética transcraneal, NSN: neurografía sensitiva nervio, NMN: neurografía motora nervio, LMC: lesión medular completa, LCC: lesión de cola de caballo.

*En fase aguda, * dependiendo de las raíces motoras S2-S4 afectadas, (alterada) 1 dependiendo de las raíces sensitivas S2S4 afectadas 
en EP la disfunción eréctil es frecuente en la atrofia multisistémica esta es prácticamente un síntoma constante. La causa es la degeneración de los sistemas autonómicos y la alteración del núcleo de Onuf. La electomiografía del esfínter anal permite detectar precozmente la denervación/reinnervación y esto permite tal vez un diagnóstico más precoz de atrofia de múltiples sistemas (14).

Mencionamos también entre las causas cerebrales de DE la esclerosis múltiple (EM) (15). En esta enfermedad no hay que olvidar el gran compromiso de la medula espinal que puede estar asociado. Por esta razón aún la prevalencia de DE es hasta el $70 \%$ de los pacientes no hay muchas peculiaridades puesto que la EM se presenta como una asociación de daño cerebral y medular con todos los posibles grados de alteración posibles. Merece la pena mencionar una posible causa de DE en EM por su posible tratamiento. Lesiones de las conexiones hipotálamo/ hipofisarias se han descrito en EM y esto está asociado con aumentos moderados de la prolactina. Este mecanismo es poco frecuente en EM pero si potencialmente interesante porque es tratable.

\section{BIBLIOGRAFÍA y LECTURAS RECOMENDADAS (*lectura de interés $y^{* *}$ lectura fundamental)}

**1. Andersson K-E, Wargner G. Physiology of penile erection. Physiol Rev 1995; 75: 191-236.

**2. Giuliano FA, Rampin O, Benoit G, Jardin A. Neural control of penile erection. Urol Clin North Am. 1995 Nov;22(4):747-66.

3. Documento de Consenso sobre Disfunción Eréctil; Foro de la Salud del Hombre en DE. Pfizer. Madrid 2002.

**4. Salinas J, Martín C, Virseda M. Bases neurofisiológicas de la disfunción eréctil. Ed Abbot Laboratories. Madrid 2003

*5. Courtois FJ, Goulet MC, Charvier KF, Leriche A. Posttraumatic erectile potential of spinal cord injured men:how physiologic recordings supplement subjective reports. Arch Phys Med Rehabil 1999;80: 1268-72.

**6. Sanchez Ramos A and Vidal Samsó J. Spanish consensus in erectile dysfunction: Specific aspects of erectile dysfunction in spinal cord injury. International Journal of Impotence Research (2004) $16, \mathrm{~S} 42-\mathrm{S} 45$.

*7. Sánchez Ramos A, Vidal J, Jáuregui ML et al. Efficacy, safety and predictive factors of therapeutic success with sildenafil for erectile dysfunction in patients with different spinal cord injuries. Spinal Cord 2001; 39: 12: 637-643.

**8. Monga M, Bernie J, Rajasekaran M. Male infertility and erectile dysfunction in spinal cord injury: a review. Arch Phys Med Rehabil 1999; 80: 1331- 1339.

**9. Di Lazzaro V, Pilato F, Oliviero A, Saturno E, Dileone M, Tonali PA. Role of motor evoked potentials in diagnosis of cauda equina and lumbosacral cord lesions. Neurology. 2004 Dec 28;63(12):2266-71.

*10. Di Lazzaro V, Oliviero A, Profice P, Ferrara L, Saturno E, Pilato F, Tonali P.The diagnostic value of motor evoked potentials. Clin Neurophysiol. 1999: 110(7):1297-307.

*11. Mauguiere F. Clinical utility of somatosensory evoked potentials (SEPs): present debates and future trends. Electroencephalogr Clin Neurophysiol Suppl. 1996: 46:27-

*12. Jea-Hun Jung, Sung-Chul Kam, Sae-Min Choi, Sung-Uk Jae, Seung-Hyun Lee, and Jae-Seog Hyun.Sexual Dysfunction in Male Stroke.Patients: Correlation Between Brain Lesions and Sexual Function. Urology 71: 99 -103, 2008.

*13. Brown RG, Jahanshahi M, Quinn N, Marsden CD (1990). Sexual function in patients with Parkinson`s disease and their partners. Journal of Neurology Neurosurgery and Psychiatry 53:480486.

*14. Hodder J (1997). Shy Drager syndrome. Axone 18:75-79.

*15. Betts CD, Jones SJ, Fowler CG, Fowler CJ. Erectile dysfunction in multiple sclerosis. Brain 1994:117;1303-1310. 